\title{
DIE PERSPEKTIVE DER ERFORSCHUNG DER RITTERORDEN IM LICHTE DER „NEUEN MILITÄRGESCHICHTE“. EINIGE BEMERKUNGEN AUS DER GESCHICHTE DES DEUTSCHEN ORDENS
}

\begin{abstract}
„Nous voyons fort bien de midi à trois heures tout ce qu'on peut voir d'une bataille, c'est-à-dire rien“.
\end{abstract}

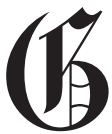
eschichte, und sogar Kriegsgeschichte, sind nicht mehr nur Schlachtengeschichte. ${ }^{2}$ Dieser abschätzige Ausdruck („Schlachtengeschichte“, „l'Histoire-bataille“) kritisierte die Manier, Geschichte in einer Art zu praktizieren, bei der es keine wissenschaftliche Quellenkritik gab. Diese Geschichte interessierte sich nur für die „Helden“ und vergaß die Strukturen. Sie konnte beschreiben, einige Vorgänge erklären, aber sie erlaubte nicht, die Schlacht als ein historisches Ereignis zu verstehen.

Seit nunmehr etlichen Jahrzehnten hat sich die sogennante „totale Geschichte" entwickelt, welche die „lange Dauer“ (la „longue durée“) von Fernand Braudel in den Blick nimmt. Diese analytische Tendenz beinflusste selbstverständlich auch die Kriegsgeschichte. Im Übrigen endet das berühmte Buch von Braudel über das Mittelmeer zur Zeit Philipps II. mit einer langen Analyse des Sieges bei Lepanto (1571). ${ }^{3}$

In der Kriegsgeschichte der letzten Jahrzehnten kann man drei Stufen erkennen. Der Krieg wird Objekt einer Geschichte, die nicht nur Militärgeschichte, sondern eine richtige Geschichte des Krieges ist. Die angelsächsischen Historiker (John France, Maurice Keen, Helen Nicholson) sprechen heute mehr von „Me-

Stendhal, Journal, vol. 5 (1811-1823), Paris 1937, S. 179.

2 Hier wird es nur eine kurze Zusammenfassung gebracht, um einige Tendenzen der Forschung vorzustellen.

3 F. Braudel, Das Mittelmeer und die mediterrane Welt in der Epoche Philipps II (deutsche Übersetzung), Frankfurt/Main 1990. 
dieval Warfare“ als von „Medieval War". Die technischen Aspekte (die Waffen, die Festungen), die Finanzierung des Krieges, die kulturellen, theologischen, politischen und rechtlichen Vorstellungen, welche die Kriegsführung beeinflussten, bleiben nicht mehr außer Betracht. Die Archäologie, die Heraldik und die Ikonographie haben unsere Kenntnis des Krieges erweitert. Zudem sind mehrere Bücher erschienen, welche die Strategie, die Taktik, die Logistik usw. in Betracht ziehen. ${ }^{4}$ Diese Forschung, die sich auf die Strukturen konzentriert, die auch die kulturellen Aspekte wie die Memoria umfasst, ist sehr verdienstvoll. ${ }^{5}$

Das Buch von John Keegan (1976) bahnte einen neuen Weg, den viele Historiker einschlugen; hier kann man Victor David Hanson, Jon Lendon, John Lynn usw. zitieren. ${ }^{6}$ Der Kern dieser Forschungen beruht auf der scharfen Kritik des erzählenden Aspekts der Kriegsberichte. Diese Historiker heben die enge Beziehung zwischen Krieg und Kultur hervor: Die Sitten und die Vorstellungen der Ritter erklären die Kriegführung. Um es kurz zu sagen: der Krieg ist ein kulturelles Phenomen. ${ }^{7}$ Diese Historiker sind aber keine Mediävisten, und 2001 schrieb Hans-Hennig Kortüm kritisch: „Die mediävistische Forschung befindet sich auf diesem wichtigen Feld noch weitgehend im Anfangsstadium".8 Dennnoch sind

4 Zum Beispiel kann man hier mehrere Historiker zitieren: J. Verbruggen, The art of warfare in western Europe during the Middle Ages (englische Übersetzung), Woodbridge 1997 (erste Aufgabe: Bruxelles 1954); P. Contamine, La guerre au Moyen Age, Paris 1994; F. Bargigia und A. Settia, La guerra nel Medioevo, Roma 2006. Dazu mehrere angelsächsische Forscher: D. Nicolle, Medieval Warfare Source Book, vol. 2, London 1996; J. France, Western Warfare in the Age of the Crusades, 1000-1300, London 1999; M. Keen, Medieval Warfare. A History, Oxford 1999; H. Nicholson, Medieval Warfare, Theory and Practice of War in Europe 300-1500, New York 2004; Medieval Warfare 1300-1450, hrsg. v. K. DeVries, London 2008; The Oxford Encyclopedia of Medieval Warfare and Military Technology, hrsg. v. C. J. Rogers, 3 Bde., Oxford 2010. Siehe auch M. Bennet, J. Bradbury, K. DeVries, Fighting Techniques of the Medieval World AD 500 - AD 1500: Equipment, Combat Skills, and Tactics, New York 2005.

5 Man weiss, daß die Mediävistik seit mehreren Jahren sich mit der Frage der „Memoria“, der „Memorialkultur“ beschäftigt. Durch die Schrift wird die Tatsache zu einem Ereignis; durch die Schrift wird sie in Gedächtnis eingegliedert. Was uns noch näher betrifft, hier muss unbedingt das Buch über den „Grossen Krieg“ der Jahren 1409-1411 zitiert werden (S. Jóźwiak, K. Kwiatkowski, A. Szweda, S. Szybkowski, Wojna Polski i Litwy z Zakonem Krzyżackim w latach 1409-1411, Malbork 2010).

6 J. Keegan, The Face of the Battle, London 1976; V. D. Hanson, The Western way of war: Infantry Battle in classical Greece, New York 1989; J. E. Lendon, Soldiers and Ghosts, New Haven 2005 (hier wird die französische Übersetzung benutzt: Soldats et fantômes. Combattre pendant l'Antiquité, Paris 2009); J. Lynn, Battle. A History of combat and culture, London 2006.

7 Im Mittelalter spielte allerdings der Krieg auch eine wesentliche Rolle in der Bildung des Adels.

8 H.-H. Kortüm, Der Krieg im Mittelalter als Gegenstand der Historischen Kulturwissenschaften. Versuch einer Annäherung, in: Krieg im Mittelalter, hrsg. v. H.-H. Kortüm, Berlin 2001, S. 13-44. 
kürzlich die Bücher von Warren Brown und Piotr Gorecki oder Malte Prietzel erschienen.?

Es gibt schlußendlich eine dritte Stufe, praktiziert zum Beispiel in Frankreich von unseren Kollegen, die über die Neuzeit arbeiten. Diese Tendenz wird durch ihren Wegbereiter Laurent Henninger als die "neue Schlacht-Geschichte“, „the new Battle-History“, bezeichnet. ${ }^{10}$ In der Mitte ihrer Überlegungen steht die konkrete, menschliche Dimension des Kampfes. Um ein einleuchtendes Beispiel zu geben: Im Buch von Olivier Chaline über die Schlacht am Weißen Berg (Böhmen 1620) wird ein Drittel des Werkes der „Erfahrung der Gewalt“ („la violence vécue“) gewidmet. ${ }^{11}$ Diese „neue Schlachtengeschichte“ will unsere Kenntnis der Realität um die Erfahrung des Kämpfers erweitern. Ihr Objekt ist natürlich die Schlacht selbst, aber ebenfalls der Mensch im Kampf. Folglich will sie den Kampf vierfacher Hinsicht untersuchen: strategisch, also die globale Kriegführung; operativ, das heißt die Analyse des Feldzuges im Kriegsschauplatz; taktisch, also der Ablauf der Schlacht; und endlich menschlich, das konkrete Erlebnis des Kampfes. ${ }^{12}$ Von diesem Standpunkt aus betrachtet, sind die Schlacht und die Belagerung einer Stadt oder einer Burg keine gegensätzliche Elemente. Während einer Belagerung gibt es viele Scharmützel, viele Ausfälle gegen die Belagerer: Man darf also nicht von einem „bewegungslosen Krieg“ sprechen. Zum Beispiel gab es zwischen dem 26. Juli und dem 19. September 1410 vor den Mauern von Marienburg mehrere Gefechte.

Das Unterschied zwischen der Schlacht und dem Feldzug scheint hier wichtig zu sein: Begriffen die mittelalterlichen Kämpfer den Krieg als eine Reihe von getrennten Schlachten oder als eine geordnete und logische Reihe von militärischen Operationen, die ein bestimmtes Ziel verfolgten? Wollten sie um jeden Preis eine „entscheidende Schlacht“ liefern, oder begünstigten sie eine Strategie, die sich auf

9 W. Brown und P. Gorecki, Conflict in medieval Europe. Changing perspectives on Society and Culture, London 2003; M. Prietzel, Kriegführung im Mittelalter. Handlungen, Erinnerungen, Bedeutungen, Paderborn 2006. Für die Geschichte des Deutschen Ordens darf man hier auch an das im Jahre 1976 erschienene Buch von Sven Ekdahl über die Banderia Prutenorum denken: Die Banderia Prutenorum des Jan Dlugosz - eine Quelle zur Schlacht bei Tannenberg 1410, Göttingen 1976.

10 Siehe den programmatischen Text von Laurent Henninger, Pour une nouvelle histoire des batailles, Cahiers du Centre d'Etudes d'histoire de la défense 9 (1999), S. 1-8. Laurent Henninger hat die Kommission "Nouvelle Histoire bataille" des CEHD (heute IRSEM: Institut de Recherches stratégiques et militaires) gegründet.

11 O. Chaline, La bataille de la Montagne Blanche (8 novembre 1620). Un mystique chez les guerriers, Paris 2000.

12 Der eigene Gesichtskreis des Kämpfers war oft sein persönnliches Überleben, oder sein Weiterleben. 
mehrere Belagerungen und Plünderungen stützte? Diese Frage darf man betreffs der polnischen Operationen im Sommer 1410 stellen.

Hier erscheint die Schlacht als ein bestimmtes Ereignis. Folglich muß man ihren Sinn bedenken. Was bringt eine Schlacht ans Licht? Was bedeutet sie? Das ist gar keine Rückkehr zur alten „Schlachtengeschichte“, sondern die Fortsetzung der modernen Kriegsgeschichte in allen ihren Dimensionen, indem der Schlacht als einem besonderen Ereignis das Hauptaugenmerk gilt. ${ }^{13}$

Die Ritterorden waren die ersten stehenden Heere des Mittelalters und ihre Mitglieder - mindestens die Ritter - waren Fachleute des Krieges. So scheint es nicht unberechtigt, ihre Geschichte bei Licht dieser Themen zu studieren. Dazu möchte ich nunmehr drei Themen vorstellen: die Schlacht als kulturelles Phenomen, die Realität des Kampfes und die Schlacht als „Chaos”. Meine Beispiele nehme ich nur aus der Geschichte des Deutschen Ordens.

\section{SCHLACHT UND KuLtur}

Die derzeitige Forschung lenkt unsere Aufmerksamkeit auf die enge Verbindung zwischen dem Krieg und der Kultur. Jede Gesellschaft betreibt den Krieg je nach ihren Techniken, ihrer Weltanschauung, ihren sozialen Strukturen, ihren Verhaltensweisen. ${ }^{14}$ Im Mittelalter gibt es wohl eine „Kriegskunst ${ }^{15}$. Man erinnert sich an die Klage der Ordensgesandten gegenüber den Polen vor dem Konstanzer Konzil (1415), die Polen „hätten den Heiden die christliche Kunst zu streiten beigebracht". ${ }^{16}$

13 Es gibt andere Forschungsrichtungen, wie zum Beispiel der anthropologische Vergleich der verschiedenen Arten und Weisen zwischen den Zivilisationen Krieg zu führen.

14 Was die Frage der Möglichkeit der technischen und taktiken Übertragungen stellt.

15 Die Quellen zeigen taktisch geordnete Verbände in den Reiterheeren.

16 Siehe Ekdahl, Die Banderia Prutenorum (wie Anm. 9), S. 11; E. Weise, Die Staatsschriften des Deutschen Ordens in Preussen im 15 Jh., Bd. 1: Die Traktate vor dem Konstanzer Konzil, Göttingen 1970, S. 107-109: [...] want die ungloubigen, von den man nu redit, itczunt obirall mit blankem harnisch, mit gerosse und andir kirchlichen geczoien mechtig dirscheinen, also das sie in reisen und hofferteger beweisunge den spitczen der cristenlichen heeren nichtes nicht geringer und gecziret werden irfunden und itczunt in der schule der cristen mit tegelicher ubunge die konst czu striten, mit welchir konst sie vorwert mogen die cristen obirwinden, haben gelart und mit iren tegelichen fleisse durch die lernunge der cristen begriffen haben manchirlei behendekeit der ritterschaft [...]. Siehe H. Boockmann, Johannes Falkenberg, der Deutsche Orden und die polnische Politik (Veröffentlichungen des Max-Planck-Instituts für Geschichte 45), Göttingen 1975, S. 14-16. 


\section{Der Krieg als „kultureller Habitus“}

Nach einem Wort Hans-Hennig Kortüms spiegelte der Krieg den „kulturellen Habitus" einer Gesellschaft wider. ${ }^{17}$ Es gibt einen kulturellen Determinismus der kriegerischen Handlungen. Hier sind die Forschungen von Otto Gerhard Oexle und seine Neubewertung des Konzepts von Kulturgeschichte sehr hilfreich: $\mathrm{Zu}$ der Kriegsgeschichte dient besonders die Analyse der Wahrnehmungs- und Deutungsmuster der Gesellschaften. ${ }^{18}$ Wie Hans Hennig Kortüm schreibt, handelt es sich bei Krieg um ein zutiefst kulturelles Phänomen. ${ }^{19}$ Die jeweilige „Kultur des Krieges" äußert sich in der spezifischen Form, in der bestimmte Gesellschaften ihre Kriege geführt haben. ${ }^{20}$

Man muß also verstehen, was der Krieg für die Zeitgenossen bedeutete und wie sie den Kampf interpretierten. Malte Prietzel fügt hinzu, dass die einzelnen Kämpfer nicht wie wehrdienstpflichtige Soldaten unserer Zeit agierten. Was uns also vernunftwidrig zu sein scheint, gehorcht den „Spielregeln“ des Mittelalters. ${ }^{21}$

17 Kortüm, Der Krieg (wie Anm. 8), S. 27. Hier soll man das Wort ,Kultur' in seinem umfangreichsten Sinn auffassen. Wie der Ägyptolog Jan Assmann geschrieben hat, ist heute „Kultur kein Kampfbegriff mehr“. Die breite Ausdehnung dieses Begriffs ist jetzt anerkennt (S. A. \& J. Assmann, Kultur und Konflikt. Aspekte einer Theorie der unkommunikativen Handelns, in: J. Assmann, D. Harth, Kultur und Konflikt, Frankfurt/Main 1990, S. 11-48, hier 35). Sicher gibt es ein Risiko mit dieser Inflationären Verwendung dieses Begriffs: das Risiko einer gewissen Beliebigkeit und einer Unschärfe.

18 Hier können die Polemologie und die Anthropologie den Historikern helfen. Es ist auch notwendig, unsere heutigen Begriffe nicht ohne Vorsicht benutzen: gelten wirklich zum Beispiel unsere Konzeptionen der Gewalt, um die mittelalterliche Gewalt zu analysieren? Das gilt auch für das Begriff von ,Krieg': gibt es ein spezifischer mittelalterlicher Krieg? Ist der mittelalterliche Krieg nur eine „blosse Fortsetzung der Politik mit anderen Mitteln“? Folglich konnte man ausschliesslich von Krieg sprechen, wo ein Staat existiert? Trotzdem, spricht man ohne Problem von Krieg für die Riterrorden, auch wenn diese Ritterordenb keinen Staat geschafft hatten - bis auf der Deutsche Orden in Preußenland.

19 Kortüm, Der Krieg (wie Anm. 8), S. 27. Er fügt hinzu: „Einer dezidiert kulturwissenschaftlichen Aufarbeitung stand in Deutschland ein restriktiver Begriff von Kultur im Sinne einer Hochkultur entgegen. Sind ,Krieg' und ,Kultur' nicht miteinander vereinbarende Begriffe? Ein weiteres Verständnis von Kultur ermöglicht es, beide Wörter in enger Beziehung zueinander zu sehen“ (S. 27). Im angelsächsischen Sprachraum wird es gesprochen von ,Kulturen des Krieges' im Sinne einer morphologischen Betrachtungsweise.

20 M. Prietzel schreibt auch: „Der Krieg im Mittelalter ist also als Teil einer Kultur zu betrachten [...]. Wie jedes andere menschliche Handeln auch, so richtet die Führung von Kriegen nicht nach ewigen, quasi-naturwissenschaftlichen Geseztmässigkeiten“. (M. Prietzel, Kriegführung (wie Anm. 9) S. 11).

21 G. Althoff, Spielregel der Politik im Mittelalter. Kommunikation im Frieden und Fehde, Darmstadt 1997. 
Ein kulturelles Phänomen kann für den Ausgang einer Schlacht entscheidend sein. Im Werk von Heinrich von Livland hat Alan Murray einen interessanten Passus aufgedeckt. Die Stadt Riga besaß im Jahre 1210 eine grosse Glocke, die campana magna, deren Klang die Heiden erschreckte. Alan Murray schreibt: „Henry states that when the pagans heard the sound of the great bell, they said that they were being eaten and consumed by the God of the Christians!" Und weiter bemerkt er, dass die Musik in den Schlachten eine wichtige Rolle spielte. Als die Heiden ein unbekanntes Instrument hörten, hörten sie sofort mit dem Kampf auf. ${ }^{22}$

Andere kulturelle Elemente können ihren Einfluss auf den Kampf ausüben. So erscheint im 14. Jahrhundert ein neues Phenomen: die breite Entwicklung zur Erteilung der Ritterschläge vor der Schlacht, die eine wichtige Veränderung der ritterlichen Kultur seit dem 12. Jahrhundert beweist. ${ }^{23}$ Die ersten Massenpromotionen fanden in den 1260er Jahren statt. ${ }^{24} \mathrm{Im}$ 14. Jahrhundert werden sie rasch üblich. Einige Jahre nach Grunwald verzeichnet der französische Chronist Enguerrand de Monstrelet solche Zeremonien vor den Schlachten bei Monsen-Vimeux (1421), Cravant (1423) oder Bulgnéville (1431). ${ }^{25}$ Jedesmal sind fast Hunderte neuer Ritter geschaffen worden. Je größer die Zahl der neuen Ritter ist, desto prestigeträchtiger wird der Ruhm der Schlacht. Unter diesen Umständen versteht man die Erhebung tausender polnischer Männer zu Rittern vor der Schlacht bei Grunwald, wenn auch diese Zahl übertrieben ist. ${ }^{26}$ Der Ritterschlag konnte auch schlechte Folgen haben, weil die durch diese Erhebung erregten neuen Ritter ohne Vorsicht handeln konnten.

Während sich die mittelalterlichen Kämpfer nach den Maßstäben der Adelsgesellschaft verhielten, scheinen die Ritterorden eine Ausnahme gebildet zu haben. Bei ihnen herrschte eine starke Disziplin, die in den Schlachten oft entscheidend

22 A. Murray, Music and cultural conflict in the Christianization of Livonia, in: The clash of cultures on the medieval Baltic frontier, hrsg. v. A. Murray, Burlington 2009, S. 293-306, besonders S. 295-299. Man erinnere sich auch an die Dudelsackpfeifer der schottischen Regimenter des 20. Jh., die in Kriegsfilmen thematisiert worden sind.

23 Prietzel, Kriegführung (wie Anm. 9), S. 247-258.

24 M. Keen, Chivalry, New Haven 1984, S. 6-8, 64-82.

25 Siehe Ph. Contamine La noblesse au royaume de France de Philippe le Bel à Louis XII. Essai de synthèse, Paris 1997, S. 282, der eine Liste über Rittererhebungen vor Schlachten und bei Belagerungen gibt.

26 Siehe die Cronica conflictus Wladislai regis Poloniae cum cruciferis. Anno 1410, hrsg. v. Z. Cielichowski, Poznań 1911, S. 22; und Jan Długosz, Annales seu Cronicae incliti regni Poloniae, hrsg. v. M. Plezia, Warszawa 1997, Bd. 11, S. 99. Nach Jean Lefèvre waren vor Azincourt (1415) 500 Männer zu Rittern erhoben (Jean Lefèvre, Chronique, hrsg. v. François Morand (Sources de l'Histoire de France 178), 2 Bde., Paris 1876-1881, hier Bd. 1, S. 259). 
war. Die Ordensritter strebten nicht nach Ruhm. ${ }^{27}$ In einer durch die adeligen Werte dominierten Gesellschaft setzten sie ihre kämpferischen Werte durch, welche die Kriegshandlungen veränderten.

Die christliche Weltanschauung beeinflusste auch die Kämpfer stark. Nach der Schlacht von Hastings (1066) sollte das normanische Heer eine Buße tun; und nach den Schlachten errichtete man oft eine Kapelle, um für das Seelenheil der Erschlagenen zu beten (sie waren gestorben, ohne dass sie bei einem Priester gebeichtet hätten). Im Mittelalter war das Gedenken an die Erschlagenen wichtiger als die Erinnerung der Schlacht selbst: Das Mittelalter kannte wahrscheinlich nicht „das institutionalisierte Erinnern an historische Ereignisse“ ${ }^{28}$ Die religiösen Stiftungen hielten vor allem das Seelengedenken wach, wenn sie auch den Besuchern des Schlachtfeldes den Sieg in Erinnerung brachten.

\section{Welche Rolle spielten die antiken Kulturmuster?}

Im Altertum war tatsächlich das Epos von Homer (die Ilias) ein Muster für die Kämpfer. Inwiefern wurde das Buch des römischen Autors Vegetius (die Epitome de re militari) benutzt oder nur von neuem erfunden ${ }^{29}$. In seiner Erzählung über Schlacht bei Grunwald schreibt Jan Długosz, dass der Deutsche Orden Ketten vorbereitet hatte, um die polnischen Ritter zu fesseln. Vielleicht ist die Begebenheit wahr, aber man liest dieselbe Geschichte in einigen antiken Quellen. In einem Artikel von 1963 verwies Danuta Turkowska auf den römischen Chronisten Iustin (3. Jahrhundert). Diese Geschichte begegnet auch schon bei Herodot. Die Spartaner griffen die Stadt Tegea an und hatten Ketten mitgenommen, um die Einwohner zu versklaven. Dennoch wurden sie besiegt und mit ihren eigenen Ketten gefesselt. ${ }^{30}$ Hatte Długosz Iustin gelesen (was Herodot betrifft, scheint es unwahrscheinlich)? Wollte Ulrich von Jungingen die polnischen Ritter wirklich versklaven?

Nützlich sind in diesem Zusammenhang die Überlegungen von Jon Lendon, der den jahrhundertelangen Einfluss der literarischen Muster auf die Kämpfer

27 Obwohl sie sich durch ihren eigenen Mantel von den anderen Kämpfern unterschieden!

28 Prietzel, Kriegführung (wie Anm. 9), S. 190.

29 M. Prietzel bemerkt zum Beispiel, daß Rahewin in seinen Gesta Friderici oft an den Jüdischen Krieg des Flavius Josephus sich lehnt (Prietzel, Kriegführung (wie Anm. 9), S. 91). Was Rahewin schildert, ist es sich wirklich geschehen? Genauso wie er es schildert? Oder hat er die Wirklichkeit geändert, um seinem Muster sich anzupassen?

30 Herodot sagt auch, daß er selbst diese im Tempel von Athena gehängten Ketten gesehen hat (Herodot. Historien, hrsg. v. J. Feix, Düsseldorf 2004, $1 \S 66$ ) 
zeigt. Er beweist, dass die Ilias eine grosse Rolle in der antiken Kriegführung spielte. ${ }^{31}$ Die technischen Fortschritte waren nicht so wichtig wie das Gedächtnis der vergangenen Schlachten und die Erinnerung an die früheren kriegerischen Traditionen. ${ }^{32}$ Jon Lendon zieht daraus den Schluss, dass die alten Griechen und Römer im Zeichen der Vergangenheit kämpften. Ob sich ein identisches Phänomen in der Welt der Ritterorden wiederholt hat, würde eine genaue Analyse unserer Quellen zeigen. Möglicherweise könnte man Einflüsse der antiken oder biblischen Urbilder entdecken. Als Peter von Dusburg die Belagerung von Christburg erzählt, schildert er die Tapferkeit eines gewissen „Sirenes“, der allein eine Brücke verteidigte: hier darf man an den römischen Helden Horatius Cocles denken. ${ }^{33}$ Man muss aber mit Vorsicht vorgehen. Peter von Dusburg war ein Priester: Seine Auffassung des Krieges konnte von der Anschauung eines Ordensritters abweichen, und die mögliche Anspielung auf einen römischen Helden konnte den Ordensrittern entgehen. ${ }^{34}$

Der Einfluß von Vegetius im Mittelalter, besonders im westlichen Europa, war unendlich groß. ${ }^{35}$ Bronisław Cetera wollte die Erzählung über die Schlacht von Grunwald bei Długosz im Lichte der Regeln der Epitoma de re militari studieren. ${ }^{36}$ Seiner Meinung nach hätten Jagiełło und die polnischen Ritter die Lehre von Vegetius angewendet. Aber wer hatte in Polen Vegetius - oder zumindest eine $\mathrm{Zu}$ sammenfassung seines Buches - gelesen? Man kennt keine polnische Handschrift dieses Textes, aber man sollte zugeben, dass dies nur ein Argument $e$ silentio ist. ${ }^{37}$

31 Hier muss man man sich erinnern, daß die Ilias die Geschichte einer zehnjähriger lang Belagerung erzählt aber, daß sie ihre Erzählung nur auf vier Tage konzentriert.

32 Anders gesagt, war die virtus so wichtig als die disciplina (Lendon, Soldats et fantômes (wie Anm. 6), S. 207). Er fügt hinzu, dass „das ganze Geheimnis der römischen Legion darin bestand, ihre Soldaten besser zu machen".

33 Petri de Dusburgk, Chronica terrae Prussie, hrsg. v. J. Wenta, S. Wyszomirski, Kraków 2007, III, 143, S. 145.

34 Bei Herrn Militzer bedanke ich mich für diese Bemerkung.

35 P. Richardot, Végèce et la culture militaire au Moyen Âge, Paris 1998; G. Lester, The Earliest English Translation of Vegetius' De re militari (Middle English Texts 2.1), Heidelberg 1988; R. J. Clifford, The Vegetian 'Science of Warfare' in the Middle Ages, Journal of Medieval History (39) 2003, 1, S. 1-19; Ch. Allmand, The De Re military of Vegetius: a Classical Text in the Middle Ages, History Today 54 (2004), 6, S. 20-25, A. Settia, De re military: pratica e teoria nella Guerra medieval (I Libri di Viella), Rome 2008. Man muss vorsichtig sein: wenn man nicht gegen die Sonne marschiert, ist es vielleicht bloss auf Grund des gesunden Menschenverstands!

36 Długoszs Beschreibung könnte das Abbild einer von Vegetius inspirierten Krieghandlung sein. B. Cetera, Bitwa Grunwaldska w świetle regut wojennych traktatu Wegecjusza, Rocznik Olsztyński (8) 1968, S. 199-231, besonders S. 202 f., 207 f., 221, 224, 226 f.

37 Im östlichen Europa kennt man nur eine Handschrift, die aus Prag stammte (XV. oder XVI. Jh., Praha, Univ. Kn., 2369, f 64-103, (durch Procopius magister Pragensis abgeschrieben). Unter den 312 bekannten mittelalterlichen Handschriften der Epitoma stammt die Mehrzahl 
Vielleicht hatten polnische Ritter einige Elemente der Epitoma während eines Aufenthalts im westlichen Europa kennengelernt? Die Hypothese von Cetera kann man also nicht beiseite schieben, wie Krzysztof Kwiatkowski feststellt ${ }^{38}$, auch wenn kein eindeutiger Beweis existiert.

Neben diesen antiken Einfluss spielten auch natürlich die christlichen Einflüsse eine große Rolle. Dazu darf man die im Mittelalter herrschenden Spielregeln des Krieges nicht vergessen. Alle diese Elemente konnten ihren Einfluss auf die Kriegsberichte ausüben. Was den konkreten Kampf betrifft, lässt es sich so jedoch nicht so klar feststellen.

\section{Die Entstehung von Stereotypen und ibre Folgen}

Der Krieg wird allmählich von der Konstruktion von Feindbildern begleitet, was konkrete - und böse! - Folgen für die Kämpfer haben konnte. Erst die Konstituierung des „Anderen“ als die eines Feindes ermöglicht oder erleichtert es, diesen Anderen zu töten. Man schafft die Begriffe von „Wir“ und „Sie“: Diese Differenzierung zwischen „uns“ und den ,anderen“ ist nicht naturgegeben, sondern sie entstammt einem Prozess kultureller Abgrenzung. Dann haben diese Stereotypen konkrete Folgen: Wenn der Gegner ein gefährlicher Feind ist, zum Beispiel ein Heide, so kann man ohne Skrupel sein Land verwüsten, seine Dörfer in Brand stecken, seine Frauen und seine Kinder versklaven. Alle diese Elemente finden wir an vielen Stellen im Werk des Peter von Dusburg oder des Heinrichs von Livland.

Die kulturelle Konstruktion des Feindbildes bestimmt teilweise die Kriegführung. Wie Hans-Hennig Kortüm bemerkt: „Der Grad der Grausamkeit, der Gewalttätigkeit mittelalterlicher Kriege, ist also primär eine Frage nach den Identitäten derjenigen, die an diesem Konflikt beteiligt sind. Nur dann, wenn eine gemeinsame Identität der Konfliktparteien gegeben ist, können auch 'Spielregeln' gelten $[. .$.$] “. { }^{39}$ Wie Krzysztof Kwiatkowski vermutet, hätten die Ordensritter während der Verfolgung nach der Schlacht bei Grunwald nicht um Gnade gebeten,

aus Italien (109) Frankreich (39), England (30) und der heutigen Deutschland (22) aus. Was die Übersetzungen betrifft, kennt man 49 französische Fassungen, 17 englische, 11 italienische und nur 2 deutsche Fassungen. Siehe Richardot, Végéce (wie Anm. 36), S. 19-28. Man kann auch eine Abschrift besitzen, ohne sie lesen zu haben.

38 Jóźwiak, Kwiatkowski, Szweda, Szybkowski, Wojna Polski (wie Anm. 5), S. 406 f. Jan Długosz kannte mehrere antiken Historiker: Sallust, Titus Livius, Florus, vielleicht Vegetius (S. W. Semkowicz-Zarembina, Elementy humanistyczne redakcji Annalium Jana Dlugosza, in: Mediaevalia $w 50$ rocznice pracy naukowej Jana Dąbrowskiego, hrsg. v. J. Garbacik, S. 236-241, 246-251). Für den letzten hat man leider kein sicheres Beweis.

39 Kortüm, Der Krieg (wie Anm. 8), S. 39. 
wenn sie gefangen genommen worden wären. ${ }^{40}$ Sie waren nämlich überzeugt, dass ihre Feinde keine Christen, sondern Heiden waren, und nur einen Christen darf man um Gnade bitten. Dies hat vielleicht die Zahl der Getöteten erhöht.

Die Analyse ist trotzdem komplizierter. Während der Zeit Ulrichs von Jungingen als Marschall des Ordens stand dieser in enger Verbindung mit Witold. ${ }^{41}$ Folglich kann man nicht behaupten, dass die Feindbilder in der Zeit der Schlacht bei Grunwald schon ganz beherrschend waren. Dagegen erwarben (sehr rasch, was bemerkenswert ist) diese Stereotypen ihre Kraft in den folgenden Jahren: Sie traten auf dem Konzil von Konstanz in die Öffentlichkeit. Die Banderia Prutenorum gibt uns ein Beispiel, wenn sie den Verrat des Herzogs Kasimir von Stettin tadelt. $^{42}$

Diese Feindbilder tragen zur Sakralisierung der Schlacht bei. In einer Schlacht geht es eigentlich um Leben und Tod, was dem Krieg einen sakralen Charakter verleiht. Das Risiko Töten und Getötet-Werden steht im Kern der Handlungen und der Vorstellungen der Kämpfer. So erweist sich der Begriff des Opfers als geeignet, um das Töten legitimieren zu können. ${ }^{43}$

\section{DER KAMPF: EINE WiRKLICHKEIT, AN DIE MAN NICHT HERANKOMMT?}

Zwischen dem Krieg und den Schriften gibt es eine dialektische Verbindung, wie dies John Lynn gezeigt hat. Die Texte verändern die Handlungen und umgekehrt. Sicher gründen sich die kriegerischen Handlungsschemata auch auf praktischen Erfordernissen, aber sie gehören zum sozialen Wissen des Heeres. ${ }^{44}$ Dazu sind die

40 Jóźwiak, Kwiatkowski, Szweda, Szybkowski, Wojna Polski (wie Anm. 5), S. 437: „Stąd bardzo prawdopodobne, że przynajmniej część braci-rycerzy i braci-sariantów o ów » pardon « podczas bitwy grunwaldzkiej wcale nie prosiła, wierząc w natychmiastowe zbawienie duszy po śmierci w walce przeciw » poganom « i ich »złym « chrześcijanskim wspomożycielom".

411404 und 1408 leistete der Deutsche Orden dem litauischen Fürsten in seinen Kriegen gegen Nowgorod und Moskau Beistand.

42 Ekdahl, Die Banderia Prutenorum (wie Anm. 9), S. 252: Non sine sua et nominis sui ac gentis et nacionis sue ac lingwarii maxima ignominia et dedecore, sacra auri fames devictus, sibi et generi suo atque prosapie opprobrium inusturus sempiternum. In viscera propria fixit gladium, in patriam propriam et regnum Polonie, a quo ipse et predecessores sui habuit esse et vivere, cum omni gente sua, que arma ferre poterat, armatus consurrexit; sed gente sua clade deleta et ipse una cum banderio et aliquibus militibus captus miserias ergastuli aliquanto tempore sustinuit, beneficio tamen Wladislai Polonie regis, ab illo tandem solutus est.

43 Kortüm, Der Krieg (wie Anm. 8), S. 28: „die Hingabe an Gott kulminiert im Opfer des eigenen Lebens, und das Töten des Feindes kann verstanden werden als Opfer für Gott“.

44 „Die Handlungen im Kampf, die Wahrnehmung der Geschehnisse und die Berichte darüber werden durch Faktoren bestimmt, die kulturell geprägt sind“" schreibt Prietzel, Kriegführung 
Schlacht und der Krieg zwei verschiedene Sachen. Der alltägliche Krieg besteht aus mehreren Elementen, die in der Schlacht nicht auftauchen und umgekehrt. ${ }^{45}$

\section{Die Schlachtberichte sind zugleich ein Tor zur Schlachtenwelt, aber auch ein Filter, ja sogar ein Schirm}

Wenn die Chronisten verbale oder gestische Provokationen erwähnen, führen sie nicht näher aus, was gesagt oder getan wurde. Malte Prietzel bemerkt zum Beispiel, daß sexuelle Anspielungen nie berichtet werden. ${ }^{46}$ Was berichtet wird, sollte das Handeln einer Seite rechtfertigen. Wie Malte Prietzel schreibt, muß man die Quellen nicht nur daraufhin untersuchen, „was sie über tatsächliches oder plausiblerweise zu vermutendes Geschehen berichten. Vielmehr rücken auch die Quellen selbst ins Blickfeld. Es muß danach gefragt werden, welche Bedeutung bestimmte Handlungen und Aussagen für die Autoren besitzen, welche Beziehungen zu anderen Vorstellungen sich ergeben, wie und warum ihre Verfasser aus den Informationen, die ihnen vorliegen, das auswählen, was sie dann tatsächlich schildern, wie sie dann von diesen Ereignissen berichten und nach welchen Kriterien sie das Berichtete gewichten und bewerten. ${ }^{47}$

In den mittelalterlichen Beschreibungen des Krieges muss man also das Gewicht der literarischen Muster bewerten. In den Chroniken kristallisieren einige Darstellungsschemata heraus, einige wiederkehrende Muster, die den zeitgenössischen Berichten zugrunde liegen. Liefern wirklich diese Wiederholungen sichere Informationen über die konkreten Vorgänge? Es ist notwendig, die literarische Erfindung, die sogenannten topoi, beiseite zu schieben, aber man darf auch nicht vergessen, dass sich die Kämpfer am Beispiel dieser Vorbilder orientierten. Jagiełło handelte wahrscheinlich wie ein rex iustus, und Jan Dlugosz porträtierte ihn als einen rex iustus. ${ }^{48}$

Die Frage der Zweikämpfe ist hier erhellend. Zweifellos waren diese Duelle ein wichtiges Element der Schlacht, auch wenn die Quellen ihre Bedeutung übertrei-

(wie Anm. 9), S. 364. Der Autor fügt hinzu: „wer nach der Kultur der Kriegführenden fragt, kann erschliessen, was Krieg für die mittelalterliche Gesellschaft bedeutete“.

45 Bei Herrn Borchard bedanke ich mich für diese Bemerkung.

46 Es ist auch plausibel, daß die adligen Kämpfer mündlich die Ereignisse verformten. „So schieben sich zwischen Geschehen und Bericht mehrere Filter" (Prietzel, Kriegführung (wie Anm. 9), S. 51).

47 Ebd., S. 18.

48 Anders ausgedrückt: es gibt Erzählungtraditionen und Handlungstraditionen, zwischen denen gewisse Einflüsse existieren oder nicht existieren können. 
ben. In der Schlachtenerzählung wird das kriegerische Geschehen individualisiert. So wollten die zeitgenössischen Ritter unbedingt einen Zweikampf gewinnen, weil der Schlachtenbericht die Normen und die Leitbilder der adligen Gesellschaft bestätigte. Dann waren sie stolz auf ihre Tapferkeit, die durch die Chronisten erinnert und den nachfolgenden Generationen überliefert wird. Sie waren sicher, dass ihre Erinnerung und ihr Ruhm lebendig bleiben würden. In einem Zweikampf können sich die adeligen Werte beweisen, und die Beschreibung der Zweikämpfe erschafft ein Bild des Krieges, welches dem Adel gefällt und die Reproduktion der adeligen Normen erlaubt. ${ }^{49}$ Der Kriegsbericht ist ein „Ruhm-Bericht“ der adeligen Gesellschaft.

Umgekehrt gibt es im tatsächlichen Kampf gewisse Elemente, welche die schriftlichen Quellen nicht genau zeigen. ${ }^{50}$ Was die Quellen uns verbergen, sind die konkreten Techniken des Kampfes, anders gesagt die Fechtkunst, das heißt genau das, was die Fechtbücher mit ihren Bildern zeigen. ${ }^{51}$ Diese Handschriften beweisen, dass die Art des Kampfes von der in den Hollywood-Filmen weit entfernt war.

Oft beschreiben die Chronisten die Schlachten in der Manier der älteren Quellen. Die Schlachtendarstellungen verändern sich zuweilen nicht so schnell wie die konkrete Realität des Kampfes. Die materiellen Veränderungen finden nicht automatisch ihren Niederschlag in den Quellen. ${ }^{52}$ Die althergebrachten Muster waren stark, ja übermächtig. Sogar die Miniaturen können uns täuschen. Die französische Historikerin Christiane Raynaud hat gezeigt, dass die Buchmalerei mehr einen antiken Einfluss als die gegenwärtige Realität widerspiegelt. „Die Bilder gestatten zumeist eben gerade nicht einen Blick auf ,the face of the battle"', schreibt Hans-Hennig Kortüm. ${ }^{53}$ Betreffs der Banderia Prutenorum bemerkt Sven

49 Jan Długosz und die Cronica conflictus berichten, daß an die Schlacht bei Grunwald ein deutscher Ritter, Luppold von Köckritz, Wladislaw Jagiełło selbst angreifen wollte (Cronica Conflictus (wie Anm. 28), S. 27; Jan Długosz, Annales (wie Anm. 28), S. 111).

50 Zum Beispiel, benutzen ziemlich oft die Ritter Kriegslisten, aber die Quellen sprechen lieber von Heldentaten.

51 Siehe den Liber de Arte dimicatoria, Ende des 13. Jh. (le Livre de l'art du combat, hrsg. v. F. Cinato, A. Surprenant, Paris 2009) oder das berühmte Codex Wallerstein, ungefähr 1470 (G. Żabinski, B. Walczak, Codex Wallerstein. A Medieval Fighting Book from the Fifteenth Century on the Longsword, Falchion, Dagger, and Wrestling, London 2002).

52 Verändern sich, fragt $\mathrm{H}$. H. Kortüm, die kulturell konditionierten Wahrnehmungen und die kulturellen Repräsentationsformen in demselben Masse und in derselben Geschwindigkeit? (Kortüm, Der Krieg (wie Anm. 8), S. 26.

53 Ebd., S. 31. Er fügt hinzu: „Bilder und Texte können als Belege fur gesellschaftliche Deutungsversuche der Wirklichkeit dienen, bilden aber Wirklichkeit niemals unmittelbar ab“. (ebd., S. 26). 
Ekdahl: „Die Zahl der verwendbaren kriegstechnischen Informationen ist recht gering, und man erhält keine Antwort auf die wichtigen Fragen nach der Einteilung der Heere in taktische Formationen und dem Verlauf des Kampfes“. ${ }^{44}$

\section{Die Zweikämpfe zwischen Realität und Idealisierung}

Spiegeln die Quellen die Realität wieder? Sie schildern die Schlacht als etwas Begreifliches, sonst wäre keine Erzählung möglich. Aber auf diese Weise lassen sie uns denken, dass der Ablauf der Schlacht rational war, was nicht ganz realistisch ist. In einer Schlacht herrschen nämlich Verwirrung, Unsicherheit, Undeutlichkeit. In seiner Erzählung wählt der Verfasser gewisse Episoden aus, um seinem Bericht eine Kohärenz zu geben. So scheint uns die Schlacht klarer zu sein, als sie tatsächlich war. Die Schlachtberichte sind Vexierspiegel.

Von diesem Standpunkt aus ist der Zweikampf der ideale Kampf. Seine Beschreibung spiegelt nicht die Verwirrung und die Gewalt der wirklichen Schlacht wieder. Sie erlaubt den Chronisten, einen eindeutigen Bericht zu verfassen. Das verwickelte und anonyme kriegerische Geschehen wird als ein persönlicher Zweikampf hochrangiger und berühmter Ritter dargestellt. Mit dieser Erzählung steigert sich auch die Dramatik des Berichtes, der sich auf gewissen Helden konzentriert und mit einem klaren Ergebnis endet. Er erscheint als eine Auseinandersetzung zwischen Gut und Böse, als ein Gottesurteil. Die Herausforderung zum Duell widerspricht nämlich nicht der Vorstellung, dass ein Duell ein Gottesurteil ist. Im Gegenteil, da ja Gott der höchste Richter sein wird, so soll man zum Duell herausfordern. Mit den Zügen eines Duells wird also die Schlacht etwas Fassbares, Heldenhaftes und Rationales. Sie erscheint nicht mehr als ein unbeschreibliches Kampfgetümmel. Der Krieg wird individualisiert und folglich weniger unmenschlich.

Waren diese Zweikämpfe in der Tat militarisch nützlich? Welches war ihr Ziel ?55 Das Duell konnte wohl entscheidend sein. Ein hochrangiger Adeliger führte seine Lehnsleute in die Schlacht. Wenn er im Zweikampf oder im Nahkampf getötet wird, ist davon auszugehen, dass ebenfalls mehrere seiner Leute getötet oder

54 Ekdahl, Die Banderia Prutenorum (wie Anm. 9), S. 156. Eine so reiche Quelle wie die Banderia Prutenorum bietet also nur ziemlich beschränkte Informationen über die Taktik und die Kriegführung.

55 M. Strickland, Provoking or Avoiding Battle? Challenge, Judicial Duel and Single Combat in $11^{\text {th }}$ and $12^{\text {th }}$ Century Warfare, in: Armies, Chivalry and Warfare. The Proceedings of the 1995 Harlaxton Symposium, hrsg. v. M. Strickland, Stanford 1998, S. 317-343. 
verletzt wurden ${ }^{56}$, oder sie wurden entmutigt und ergriffen die Flucht. Meistens gab es im Mittelalter keine Kommandostruktur, keine Befehlskette, welche die bösen Folgen dieses Todes behindern konnte. Der Tod eines Fürsten war folglich oft entscheidend, weshalb die Quellen ein solches Ereignis berichten, und nicht nur, weil sie ihren Bericht auf einige adelige Figuren konzentrieren.

Das Duell enthält auch ein großes Risiko. Ein Fürst, ein König darf sich nicht in Gefahr begeben. ${ }^{57}$ In der Schlacht bei Grunwald hindern die polnischen Ritter den König Jagiełło daran, gegen seine Feinde vorzuspringen. Moderne Staatsraison setzte sich gegen die gewöhnliche adlige Tapferkeit durch. Alles in allem besaß der Zweikampf eine riesige Bedeutung für die Praxis des Krieges und für die Vorstellung von Schlachten und von vorbildlichen Rittern.

\section{Dennoch muss man nach den Realien suchen}

Das Interesse konzentriert sich noch zu einem Gutteil auf die Realien des Krieges. Der Standort der Sonne (Crécy, 1346) und die Topographie des Schlachtfeldes sind wichtige Elemente, die helfen, den Ablauf einer Schlacht zu verstehen, wie Sven Ekdahl in seinem Artikel über den Aufmarsch und die Aufstellung der Heere bei Grunwald gezeigt hat. ${ }^{58}$ Die Waffen, die Zahl der Erschlagenen, die Ausrüstung und die Kriegführung bleiben der Kern der historischen Analyse.

Nehmen wir das Beispiel der Flucht der Litauer während der Schlacht bei Grunwald. Entweder handelte es sich um eine echte Flucht, wie S. M. Kuczyński meinte, oder um eine List der Litauer, wie Sven Ekdahl vermutet. ${ }^{59}$ In diesem Fall

56 „Hinter jedem Namen eines Fürsten, der von einem Chronisten als gefallen gemeldet wird, ist also im Allgemeinem eine Anzahl weiterer Toter und Verletzer - und zwar wiederum Männer, die zum effektivsten Teil des Heeres gehörten, nämlich schwer gepanzerte Reiter" (Prietzel, Kriegführung (wie Anm. 9), S. 128).

57 W. Goez, Über Fürstenzweikämpfe im Spätmittelalter, Archiv für Kulturgeschichte (49) 1967, S. 135-163; R. Schneider, Zweikampf von Königen - statt blutiger Kriege?, in: Regionen Europas Europa der Regionen. Festschrift für Kurt-Ulrich Jäschke zum 65. Geburtstag, hrsg. v. P. Thorau, S. Penth, R. Fuchs, Köln-Weimar-Wien 2003, S. 21-32.

58 S. Ekdahl, Aufmarsch und Aufstellung der Heere bei Tannenberg/Grunwald (1410). Eine kritische Analyse, in: Krajobraz Grunwaldzki w dziejach polsko-krzyżackich i polsko-niemieckich na przestrzeni wieków. Wokót mitów irzeczywistości, hrsg. v. J. Gancewski, Olsztyn 2009, S. 31-104.

59 S. M. Kuczyński, Wielka wojna z Zakonem krzyżackim w latach 1409-1411, Warszawa ${ }^{4} 1980$, S. 294-295; S. Ekdahl, Die Flucht der Litauer in der Schlacht bei Tannenberg, Zeitschrift für Ostforschung (12) 1963, 1, S. 11-19. Diese List findet man oft in Westeuropa. Im Jahre 1374 zum Beispiel verwenden die Engländer diese Falle gegen Walleran III vom Luxemburg (Jean Froissart, Chroniques, hrsg. v. S. Luce, 25. Bde., Paris 1869-1879, hier Bd. 8, S. 186: Sitos que li Englès furent venus jusques à yaus, ils fisent leur moustre, et tantost se misent au retour pour revenir 
wollten die Litauer die Aufstellung des Deutschordensheeres stören. Dennoch bleibt eine Frage. Peter von Dusburg erzählt viele Male, dass die Heiden den Rittern eine solche Falle stellten, indem sie eine Flucht fingierten. ${ }^{60}$ Weshalb wurden dann die Ritter an die Schlacht bei Grunwald so unvorsichtig? Konnten sie wirklich diese Taktik ignorieren? Der linke Flügel des Ordensheeres war den Söldnern und den Gästen anvertraut. Diese Kämpfer waren nicht gewohnt, gegen die Litauer und die Tatarer zu kämpfen, und man kann vermuten, dass sie diese List nicht kannten. Das Risiko war so größer, dass bei diesen Söldnern eine relative Disziplinlosigkeit herrschte.

Die konkrete Realität des Kampfes scheint dennoch meistens unbekannt zu bleiben. Was wissen wir zum Beispiel von den Empfindungen der Kämpfer? Ihre emotionalen Komponenten - Gier, Hass, Angst - sollen in den Vordergrund gestellt werden. Dank der schriftlichen, archäologischen und ikonographischen Quellen können wir gewisse Hypothesen aufstellen, aber wir haben fast keine direkte Aussage.

\section{IsT DIE SCHLACHT EIN INKOHÄRENTES EREIGNIS?}

\section{Die Schlacht als Chaos}

Hier liegt ein Leitmotiv der „neuen Schlachtengeschichte“: Wie der Held von Stendhal (Fabrice del Dongo in der Schlacht von Waterloo ${ }^{61}$ ) wird der Kämpfer fast blind. Als dynamischer Vorgang ist die Schlacht eine chaotische, durch Lärm, Verwirrung und extreme Schnelligkeit beherrschte Welt ${ }^{62}$, eine Welt, die aus Erschütterung und Abbruch besteht, wo die Gewalt unbezwingbar ist und wo geschickte Bewegungen schwer sind. In einer Schlacht verlieren die Kämpfer ihre empfindlichen und rationellen Orientierungspunkte - erst nach der Schlacht nehmen sie wieder allmählich Vernunft an.

Eine Schlacht ist eine stark klangvolle Welt. Zuerst handelt es sich um verständliche Klänge: die Ansprache, die Gesänge und die Schlachtrufe. Bei Grunwald hat das polnische Heer Bogurodzica gesungen, während die Ordensritter Christ ist erstanden sangen. Diese Gesänge elektrisierten die Kämpfer (sie stärken auch das Zwerchfell, mithin die respiratorische Kapazität). Aber schnell wird die Schlacht

à leur compagnons, qui les attendoient au lonch de le haie en très bonne ordonnance, leurs archiers pardevant yaus).

60 Siehe zum Beispiel, Dusburgk (wie Anm. 34), III, 138, S. 141.

61 Stendhal, La chartreuse de Parme, Paris 1864, Kap. III, S. 42 f.

62 Was die Historiker des Altertums (V. D. Hanson, J. Lendon) gut gezeigt haben. 
zum klangvollen Chaos, wo sich der Zusammenstoß der Waffen, die Kanonade, die Schmerzgebrüll der Kämpfer, der Galopp und das Gewieher der Pferde mischen. Der Kämpfer wird plötzlich fast taub, und er gerät in eine heftige Welt, die ein Trauma hervorrufen kann.

Er wird auch blind, besonders des Staubs wegen. Die Abläufe gehen so schnell, dass er nichts genau erblicken kann. Dazu kann er nur einen geringen Ausschnitt der Schlacht sehen. Als Ganzes bleibt das Schlachtfeld unsichtbar. Wer nicht auf einer Höhe steht und eher aus der Ferne zusieht, kann den Ablauf der Schlacht nicht begreifen. Dagegen fühlen die Kämpfer wohl, ob ihre eigene Linie vorrückt oder ob sie vor dem Feind zurückweicht. Sie waren also nicht total empfindungslos.

Im Gegenzug ermöglichen es die Banner, eine gewisse Ordnung zu schaffen. Um diese Banner herum können sich die Kämpfer zusammenschließen. Mit ihrer Hilfe konnte man Befehle und Signale geben. Sie waren sichtbare Zeichen, die eine konkrete und psychologische Hilfe boten. Ebenso geben die Musikinstrumente (Trompeten, Posaunen, Pfeifen) den Kämpfern notwendige Informationen: „Wenn der aufwirbelnde Staub die Sicht auf dem Schlachtfeld behinderte, waren die Töne das einzige, wonach man sich richten konnte“.63

Die Taktik und die Kampfkunst spielten auch ihre Rolle: Die Schlacht war keine absolute Unordnung. Klaus Militzer schreibt: „Es gibt sicher mehr Beispiele für ein rational-taktisches Verhalten in der Schlacht als für unsinnige Abweichun-

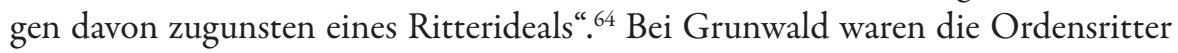
in drei verschiedene Kontingente eingeteilt. Damit eine solche Aufstellung wirksam wurde, mussten die Ritter gemeinsam trainieren. Vielleicht war dies leichter für die Ritterorden als für die anderen Kämpfer? Leider gibt es keine Beweise für das Exerzieren von Verbänden der Ordensritter in Preussen. ${ }^{65}$ Übrigens befand sich der Deutsche Orden jedes Jahr im Krieg, was eine gute Ausbildung war.

Obwohl die Schlacht so verworren zu sein scheint, besteht sie dennoch aus Truppenbewegungen und mehreren individuellen Kämpfen, die verständlich und

63 Ekdahl, Die Banderia Prutenorum (wie Anm. 9), S. 17. Wohl berühmt ist auch das Beispiel Jagełłos vor der Schlacht bei Grunwald, der seinen Heeren die Feldrufe »Kraków« und »Wilno« gab. Der König befahl auch den polnischen und litauischen Soldaten, sich mit Strohbüscheln als Erkennungszeichen zu versehen.

64 K. Militzer, Das Problem der zwei Schwerter in der Schlacht bei Tannenberg, in: Rechtsverständnis und Konfliktbewältigung. Gerichtliche und außergerichtliche Strategien im Mittelalter, hrsg. v. S. Esders, Cologne, Weimar, Vienne 2007, S. 379-389.

65 Aber das Schweigen der Quellen ist kein Beweis, daß ein solches Exerzieren nicht vorkam, s. Ekdahl, Die Banderia Prutenorum (wie Anm. 9), S. 15. Vielleicht ist es sogar ein Beweis, daß dieses Üben als selbstverständlich angesehen wurde. 
sichtbar sind. In diesem Chaos gibt es gewisse unchaotische Elemente: Letztlich liegt alles hier am Maßstab. ${ }^{66}$

\section{Die schreckliche Realität des Kampfes}

Man soll keine Ästhetisierung oder Romantisierung des Krieges betreiben: Eine Schlacht ist kein lyrisches Schauspiel. Sicher ist der Krieg ein Akt des Rechts (der Begriff des bellum iustum war grundlegend), aber er bewahrt immer seine ,Spezifizität': töten und getötet werden. Selbst wenn ein Krieg „,ritterlich“ geführt wird was zweifelhaft ist -, verliert er nicht seine Gewalt und seinen Schrecken. Unsere Quellen sprechen selten von den Infanteristen, und man kann zum Beispiel nicht glauben, dass das polnische Heer bei Grunwald fast keinen Mann verloren hätte, wie Wladislaw Jagieło geschrieben hat. ${ }^{67}$ Tatsächlich hatte dieses Heer nur einige adelige Männer verloren.

Man darf also die schrecklichen Folgen des Krieges nicht vergessen: die Verwüstungen, die Verletzungen, die Verstümmelungen, die abscheulichen Gerüchte des Schlachtfeldes. So sollte man jeden Ästhetizismus des Krieges vermeiden, wenngleich unsere Quellen ab und zu eine idealisierte Vorstellung liefern. Hier darf man trotzdem bemerken, dass die Ikonographie sogar oft ungeschminkt grausam ist.

Herrscht dennoch über den Krieg eine unkontrollierte Gewalt? Clausewitz hat geschrieben: „Der Krieg ist also ein Akt der Gewalt“ ${ }^{68}$ Die Gewalt existiert: Es ist ein Antun und auf der Seite des anderen ein Erleiden. ${ }^{69}$ Im Krieg tötet man, im

66 Der Wechsel des Masstabs scheint entscheidend zu sein: er verändert unseres Verständnis des Phenomens und erlaubt uns, eine globale Vision der Schlacht zu ergreifen.

67 Paucis valde de nostris communibus, nullis nobilibus interfectis, Brief des Königs Ladislas Jagiełło an den Bischof von Poznań, in: Scriptores Rerum Prussicarum, hrsg. v. Th. Hirsch, Bd. III, Leipzig 1866, S. 427; Jan Długosz, Annales (wie Anm. 27), Bd. 11, S. 121 (Supputatione autem facta compertum duodecim tantummodo notabiles milites de exercitu regio cecidisse).

68 Kortüm, Der Krieg (wie Anm. 8), S. 29.

69 Hier kann man die Definition von Heinrich Poppitz akzeptieren: „Gewalt meint eine Machtaktion, die zur absichtlichen körperlichen Verletzung anderer führt" (H. Poppitz, Phänomene der Macht. Autorität-Herrschaft-Gewalt-Technik, Tübingen 1986, S. 73). Siehe auch das Werk von Carl Schmitt: „Die Begriffe Freund, Feind und Krieg erhalten ihren realen Sinn dadurch, daß sie insbesondere auf die reale Möglichkeit der physischen Tötung Bezug haben und behalten. Der Krieg folgt aus der Feindschaft, denn diese ist seinsmässige Negierung eines anderen Seins. Krieg ist nur die Äusserste Realisierung der Feindschaft“ (Der Begriff des Politischen, Hamburg ${ }^{1}$ 1927; hier zitiet Hamburg ${ }^{3}$ 1933, S. 15). 
Krieg wird man verletzt. Wenn auch einige Kriege kontrolliert werden, wenn auch Spielregeln für beschränkte Gewaltanwendung existieren, wie es Gerd Althoff für das Hochmittelalter gezeigt hat, gibt es keinen Krieg ohne Gewalt. ${ }^{70}$

Aber Gerd Althoff spricht besonders von Konflikten zwischen den Edelleuten, das heisst von innergesellschaftlichen Konflikten. Die Lage ändert sich vielleicht für die, interstaatlichen' Kriege. Der Begriff eines gewaltloses Krieges scheint nicht zutreffend zu sein, weder für die Kriege des Deutschen Ordens gegen die Heiden, noch für den großen Krieg von 1409-1411, der keine Fehde war, sondern ein Krieg zwischen zwei Staaten. Dort hat man getötet und wurde man getötet. Trotzdem gab es noch gewisse Spielregeln, wie die Klage des Deutschen Ordens beim Konzil von Konstanz gegen Witold zeigt, gemäß der dieser Fürst drei Ordensritter enthaupten lassen hatte. ${ }^{71}$

Nochmals wollen wir Clausewitz zitieren: „Ohne Töten aus Feindschaft gibt es keinen Krieg“. Im Krieg beobachtet man oft eine „Entgrenzung“ der Gewalt: hier kann man an die blutige Eroberung der Stadt Gilgenburg denken. Man muss die Formen prüfen, in denen sich die Gewalt konkretisiert. ${ }^{72}$ In den Kriegen zwischen den Christen und den Heiden herrscht eine besondere Grausamkeit. Wir alle kennen gewisse Stellen bei Peter von Dusburg, wenn auch einige aus früheren Quellen stammen (wie die Geschichte des Mannes, der mit seinen Gedärmen an einem Baum festgebunden wurde: dieselbe Geschichte findet man in der „Geschichte des ersten Kreuzzugs“" von Robert dem Mönch). ${ }^{73}$

70 Siehe zum Beispiel Gerd Althoff: „[...] in mittelalterlichen Konflikten bestand jederzeit die Möglichkeit, die Gewaltanwendung durch Unterwerfung zu beenden“" (G. Althoff, Schranken der Gewalt. Wie gewalttätig war das 'finstere Mittelalter'?, in: H. Brunner, Der Krieg im Mittelalter und in der Frühen Neuzeit: Gründe, Begründungen, Bilder, Braüche, Recht (Imagines Medii Aevi 3), Wiesbaden, 1999, S. 1-23.

71 Et eciam quod Wytowdus post proximum bellum captivitatis tribus fratribus ordinis inter alios satis strenuos et nobiles eos post captivitatem huiusmodi decapitare fecit, quod tamen hucusque fuit inter christianos contra regula militarem (Codex epistolaris Vitoldi Magni Ducis Lithuaniae, 1376-1430, hrsg. v. A. Prochaska (Monumenta medii aevi historica 6, 2), Kraków 1882, Pars 2 , Appendix, S. 1024-1033, hier 1033).

72 Mithin dürfen nicht die körperlichen Aspekte von Krieg und Gewalt aus dem Vordergrund gedrängt werden.

73 Dusburgk, Cronica, (wie Anm. 34), III, 66, S. 99: Prutheni ligaverunt eum vivium per manus ad arborem et excisum umbilicum ventris sui, cui adhaerebat viscus, affixerunt arbori, quo facto plagis multis compulerunt eum, ut circumiret arborem, quousque omnia viscera ipsius arbori adhaeserunt [...]. Siehe den Text von Robert le Moine: Et quos eis placet turpi occubitu mulctare, umbilicum eis perforant, caput vitaliorum abstrahunt, ad stipitem ligant, et sic flagellando circumducunt, quoadusque extractis visceribus solo prostrati corruunt (Robert le Moine, Histoire de la Première Croisade, hrsg. v. F. Guizot (Collection des mémoires relatifs à l'Histoire de France), Paris 1824, Livre I, Chap. 1, S. 302). 


\section{Die Schlacht als Urbild des Ereignisses}

Hier liegt schlussendlich das Postulat der „neuen Schlachtengeschichte“: durch ihre Dichte ist die Schlacht das „Urbild des Ereignisses“ (L. Henninger), das Ereignis schlechthin. Der französische Historiker E. Leroy-Ladurie hat zwischen zwei verschiedenen Ereignissen unterschieden: einerseits dem „Matrizen-Ereignis“, das eine ganze Reihe von bedeutenden Folgen gehabt hat, und andererseits dem „Lupen-Ereignis", das die inneren Strukturen einer Gesellschaft ans Licht bringt. ${ }^{74}$ Bei Licht besehen, scheint die Schlacht bei Grunwald gleichzeitig eine Matrize und eine Lupe zu sein, obschon auch sie nicht ganz entscheidend war, wie die Fortführung des Krieges (die Belagerung der Marienburg, die Schlacht bei Koronowo) und die Klauseln des Ersten Thorner Friedens zeigen.

Was bringt diese „neue Schlachtengeschichte“ wirklich Neues? Vielleicht die Manier, viele Maßstäbe zu mischen, um die Schlacht als ein entscheidendes Ereignis zu begreifen. Sie erinnert uns auch, dass die eigene Erfahrung des Kämpfers ein grundlegender Bestandteil des Krieges ist. Umgekehrt kann vielleicht die Geschichte der Ritterorden der allgemeinen mittelalterlichen Kriegsgeschichte nützen.

74 „[... Je prendrais pourtant bien volontiers le braudélisme à la carte plutôt qu'au menu: soit l'histoire des petites gens et de la longue durée, mais aussi la réhabilitation de l'é véne $\mathrm{m}$ e $\mathrm{n} \mathrm{t}-\mathrm{m}$ at rice, qui engendre une série de conséquences bénéfiques ou non, se répercutant sur plusieurs siècles, comme dans le cas de la Peste Noire (1348) ou de la mort de Louis XIV. Réhabilitons aussi l'évé n e m en t - lo u pe : il permet de déchiffrer les structures profondes d'une société, telle celle de Paris à l'époque de la Saint-Barthélémy (1572)“, Le Monde poche, 15 avril 1995, p. VI (ich unterstreiche). 
